\title{
DESARROLLO DE ESTACAS DE Prunus salicina INJERTADAS in situ EN DIFERENTES SUSTRATOS CON LOMBRIHUMUS ${ }^{1}$
}

\author{
Anacleto González-Castellanos ${ }^{2}$,Graciela Noemí Grenón-Cascales², Luis Miguel Vázquez-Garcíaª , \\ Araceli González-Nicanor ${ }^{2}$,Amalia Pérez-Hernández ${ }^{2}$
}

\begin{abstract}
RESUMEN
Desarrollo de estacas de Prunus salicina injertadas in situ en diferentes sustratos con lombrihumus. El objetivo del presente trabajo fue determinar el efecto de sustratos con lombrihumus en el desarrollo de plántulas de ciruelo en vivero. Se estacaron e injertaron in situ secciones de Prunus salicina var. Mariana 2624 con P. salicina var. Methley sembrados en sustratos de lombrihumus. El trabajo se realizó en la Facultad de Ciencias Agrícolas de la Universidad Autónoma del Estado de México en condiciones de campo, de diciembre de 2002 a enero de 2003. Se empleó un diseño experimental completamente aleatorizado con arreglo bifactorial con cuatro tratamientos y cuatro repeticiones. Los factores en estudio fueron época de estacado e injertación y diferentes tipos de sustratos. Las variables en estudio fueron: longitud de planta, diámetro del tallo, número de nudos, porcentaje de prendimiento, número de hojas por planta, área foliar, perímetro y longitud de la hoja. La fecha óptima para la realización de estacado e injertación in situ en condiciones de campo para Toluca, México, fue en enero. El mayor porcentaje de prendimiento se observó con el sustrato formado por: $20 \%$ de turba (suelo de azolve), $30 \%$ de perlita, $50 \%$ de lombrihumus. Mediante esta tecnología se obtuvieron plantas de ciruelo ( $P$. salicina var. Methley) en nueve meses.
\end{abstract}

Palabras clave: Área foliar, Eisenia andrei.

\begin{abstract}
Development of Prunus salicina cuttings grafted in situ in different susbtrates containing lombrihumus. The objective of this study was to determine the effect of substrates with lombrihumus on the development of plumb cuttings growing in a nursery. Sections of Prunus salicina var. Mariana 2624 were grafted on con P. salicina var. Methley and planted on substrates containing lombrihumus. The work was conducted at the Faculty of Agricultural Sciences, UNAM, México, under field conditions, from December 2002 to January 2003. A completely randomized was employed, with factorial arrangement with four treatments and four replications. The factors studied were the time of cutting and grafting and the different types of substrates. The variables measured were plant length, cutting diameter, number of internodes, percent successful grafting, number of leaves per plant, leaf area, perimeter and length. The optical time for cutting preparation and grafting in situ under field conditions in Toluca, México, was January. The largest grafting success was obtained with susbtrate composed of $20 \%$ peat (azolve soil), $30 \%$ perlite, and $50 \%$ lombrihumus. With this technology, fully developed plumb plants can be obtained in nine months.
\end{abstract}

Key words: Leaf area, Eisenia andrei.

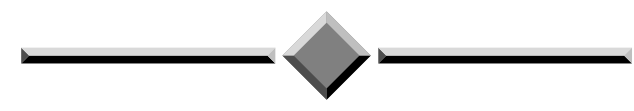

\footnotetext{
1 Recibido: 8 de junio, 2009. Aceptado: 3 de octubre, 2011. Tesis de grado del primer autor, Maestro en Ciencias Agropecuarias y Recursos Naturales. Universidad Autónoma del Estado de México.

2 Facultad de Ciencias Agrícolas, Universidad Autónoma del Estado de México. Instituto Literario No. 100. Col. Centro, Toluca, Estado de México, México.CP 5000. anacleto_glez@hotmail.com; grace_grenon@hotmail.com; 1muvg@uaemex.mx; aragonicanor@hotmail.com; mayofce@yahoo.cpm.mx
} 


\section{INTRODUCCIÓN}

En los últimos años dos problemas aquejan a la humanidad, por un lado el exceso de residuos derivados de las diferentes actividades humanas y por otro, la necesidad de producir alimentos naturales. En el primer caso los residuos orgánicos pueden ser transformados en sustancias útiles para ser incorporadas al suelo y servir como abono a las plantas que se desarrollan sobre ellos, y se obtiene en muchos casos un mayor vigor.

En la fruticultura la propagación de material vegetativo a partir de estacas, es un método muy usado para obtener plantas de manera rápida y efectiva, por la facilidad de las estacas de formar callo basal y la emisión de raíces. Este método permite obtener gran cantidad de plantas en un lapso corto, si se conoce la mejor época del año para proceder a esta actividad.

Está demostrado que la aplicación de materia orgánica a los suelos de cultivo o a los sustratos preparados para macetas o viveros, permiten que la planta obtenga nutrimentos de forma apropiada, ya que ésta favorece la capacidad de intercambio catiónico entre la mezcla y el sustrato (Grenón 2001).

En la producción de plantas terminadas en viveros (plantas de calidad comercial) se procede de la siguiente forma: se enraiza la estaca, luego se realiza el injerto y su posterior mantenimiento, proceso que lleva de 18 a 24 meses. Se ha propuesto que los clones con mejor respuesta para formar nuevos individuos de ciruelo son el Mirabolano y Mariana 2624 (Hartmann y Kester 1999, García 2000). El comportamiento de las estacas de $P$. salicina cuando se colocan a enraizar es muy variable dependiendo del cultivar, los reguladores de crecimiento, las épocas de realización, ambientes y tipos de sustratos entre otros (Dutra et al. 2002).

La riqueza del lombrihumus se debe a que: Le proporcionan al suelo mayor porosidad y aireación, mejorando también la infiltración y favoreciendo el desarrollo radical; los nutrientes que las plantas necesitan se van liberando gradualmente, pues al mantener al $\mathrm{pH}$ dentro de un rango cercano a la neutralidad 6 a 7 (con gran poder buffer) le permite una mayor solubilidad. El tenor de microelementos: $\mathrm{Cu}, \mathrm{Mn}, \mathrm{Mo}$ y $\mathrm{Zn}$ es elevado $\mathrm{y}$, contienen los mismos microorganismos benéficos que tiene el suelo, pero en mayor cantidad, destacándose los que transforman la celulosa y los que intervienen en la asimilación de nitrógeno y fósforo (Mirabelli 1995).
Se encontró que el humus de lombriz contiene gran cantidad de enzimas y bacterias que facilitan la asimilación de los nutrimentos por las raíces, al tiempo que impiden que éstos sean lavados por el riego, manteniéndolos por más tiempo en el suelo (De Sanzo y Ravera 1999).

La baja reserva de nutrientes es el principal causante de niveles sub-óptimos del área foliar y de muchas otras áreas. Igualmente se ha demostrado que una baja disponibilidad de agua en el suelo, altos déficits de presión de vapor, y temperaturas elevadas afectan en forma negativa la producción y/o retención del área foliar. La variación en la producción por unidad de área foliar (generalmente definida como la inclinación de la producción de madera - relación de área foliar y que es conocida como crecimiento o eficacia del área foliar) también pueden contribuir a su variación. La eficacia en el crecimiento puede variar debido a las diferencias en eficacia fotosintética, respiración, y división de varios componentes de la biomasa (Hartmann y Kester 1999).

Se ha evaluado la aplicación de lombrihumus como alternativa para la fertilización de plántulas de duraznero en vivero, destacando el aumento en el área foliar, peso seco de la parte aérea, de raíz y el número de brotes respecto a las plantas testigo (Rodríguez et al. 1992). En algunos trabajos, los mejores resultados de enraizado pueden ser obtenidos al inicio o final de la dormancia (Szecsko et al. 2001).

En P. salicina usado como portainjerto y aplicando ácido indolbutírico como enraizador, se obtuvo que el porcentaje de prendimiento más alto fue en los cortes realizados el 15 de diciembre (Sandhawalia 1996).

La Fundación Salvador Sánchez Colín CICTAMEX, S. A. Coatepec Harinas, Estado de México, realizó un trabajo de propagación de la macadamia por injertación y estacado, la injertación se realizó en plántulas de macadamia (Macadamia integrifolia) de nueve meses de edad provenientes de semilla y el estacado en cinco cultivares de la misma especie, estas dos actividades se realizaron de manera independiente y no in situ, además de que no se empleó ningún medio controlado para su desarrollo, sino las condiciones de campo propias de la zona de estudio (Cruz et al. 1998).

El objetivo del presente trabajo fue determinar el efecto de sustratos con lombrihumus en el desarrollo de plántulas de ciruelo en vivero. 


\section{MATERIALES Y MÉTODOS}

Este trabajo se llevó a cabo en la Facultad de Ciencias Agrícolas de la Universidad Autónoma del Estado de México, en el invierno del 2002.

Las instalaciones se encuentran ubicadas en el Campus Universitario El Cerrillo, localizado a 18 $\mathrm{km}$ de la ciudad de Toluca, México, a $19^{\circ} 26^{\prime}$ Latitud Norte y $99^{\circ} 43^{\prime}$ Longitud Oeste, a una altitud de 2656 metros sobre el nivel del mar. El clima imperante en el área de estudio es el templado con lluvias en verano (García 1988). La temperatura media anual es de $12,6^{\circ}$ $\mathrm{C}$, con incidencia de heladas en el periodo invernal. La precipitación media anual es de $878,4 \mathrm{~mm}$.

La elaboración de la composta aeróbica se preparó con el método de montículo con adición de inóculo. Para ello se emplearon 100 litros de agua, y se disolvió $5 \mathrm{~kg}$ de azúcar, $5 \mathrm{~kg}$ de urea y 100 gramos de levadura de cerveza seca, mezclándose de forma homogénea (Grenón 2001).

Los materiales a compostear fueron $20 \%$ de residuos de rastrojos de maíz molido, $40 \%$ de estiércol equino y $40 \%$ de residuos verdes (restos de pasto verde recién cortado, malezas y restos de flores y hojas de alcatraz) y se dispusieron sobre suelo libre de materiales en una superficie de $4 \mathrm{~m}^{2}$. De manera intercalada se fueron colocando el rastrojo previamente molido y húmedo, seguidos del estiércol, luego una capa con los materiales verdes, este procedimiento se realizó hasta alcanzar una altura de un metro. En cada una de las capas de los materiales composteados, se distribuyó con regadera manual el inóculo preparado con anterioridad.

Se fermentaron de forma aeróbica: rastrojo de maíz, estiércol equino y diversos residuos vegetales. Cuando la fermentación finalizó el producto obtenido (composta) se le suministró a la lombriz roja californiana (Eisenia andrei) para la producción de lombrihumus (Guerrero 1998, Grenón 2001, Grenón y González 2008). Se consideró que la fermentación había terminado cuando las temperaturas de la composta y del ambiente fueron las mismas; la composta alcanzó una coloración marrón oscura, pasó por un tamiz del número tres $(5 \mathrm{~mm})$ y tenía un olor a tierra húmeda (Grenón y González 2008).

Se prepararon bancales de $0,8 \times 5 \times 0,4$ metros, donde se dispuso parte de la composta y un kilo de lombrices por metro cuadrado, aplicándose una capa de
$5,0 \mathrm{~cm}$ de espesor de la composta cada semana (Grenón y González 2008). El lombrihumus se extrajo a los tres meses, tiempo en el que se recuperaron las lombrices.

Con el objeto de preparar las mezclas de suelo donde se colocarían las estacas, se tomó el lombrihumus, turba (suelo de azolve) y perlita, y se prepararon los sustratos volumen a volumen (litro a litro) analizándose en el Laboratorio de Suelos de la Facultad de Ciencias Agrícolas de la UAEMéx.

Las fechas de estacado e injertación "in situ" fueron el 15 de diciembre del 2002 y 15 de enero del 2003, como Factor "A" y cuatro sustratos como el Factor "B", del diseño experimental.

Se seleccionaron estacas de $P$. salicina var. Mariana 2624 de $25 \mathrm{~cm}$ de longitud y 0,4 a $0,6 \mathrm{~mm}$ de diámetro (Calderón 1998) como portainjerto, se les adicionó un enraizador en talco (ácido indol -3-butírico) en el extremo basal y en el apical, y por último, se les colocó la yema de P. salicina var. Methley como injerto, con base en el enchapado lateral (yema con astilla) en un estadio intermedio entre ojo dormido (yema en crecimiento activo) y ojo venido (yema a finales del reposo). El enraizado y estacado simultáneo fue el segundo factor de la investigación.

Una vez injertadas las estacas se colocaron en las mezclas previamente preparadas (Cuadro 1).

Cuadro 1. Composición de los sustratos utilizados como tratamientos para la producción de plántulas de ciruelo en invernadero en las dos fechas: 15 diciembre 2002 y 15 enero 2003. Facultad de Ciencias Agrícolas de la Universidad Autónoma del Estado de México (FCA-UAEM), México. 2003.

\begin{tabular}{cccc}
\hline Sustrato & \% Turba & \% Perlita & $\begin{array}{c}\text { \% Lombri- } \\
\text { humus }\end{array}$ \\
\hline A & 60 & 30 & 10 \\
B & 40 & 30 & 30 \\
C & 20 & 30 & 50 \\
D (Testigo) & 70 & 30 & 00 \\
\hline
\end{tabular}

El tratamiento testigo se consideró de esa forma ya que los viveristas utilizan esta mezcla cuando propagan sus plantas. A los cuatro tratamientos se les adicionó una décima parte de cisco (polvo de carbón). 
Estos tratamientos se ensayaron en dos fechas que correspondieron al 15 de diciembre de 2002 y 15 de enero de 2003, Factor "A".

El diseño experimental utilizado fue aleatorizado con arreglo bifactorial con cuatro tratamientos y cuatro repeticiones. Se manejó como unidad experimental diez estacas injertadas in situ por tratamiento y repetición.

A los nueve meses de la preparación de estacas e injertos se procedió a la medición de las siguientes variables: Longitud de planta, desde el suelo (cuello del vegetal) al ápice caulinar (medidas en $\mathrm{cm}$ ); diámetro del tallo, considerado a los $30 \mathrm{~cm}$ del cuello (medidas en $\mathrm{cm}$ ); nudos: número de nudos; porcentaje de prendimiento: número de plantas logradas por ciento; hojas por planta: número de hojas. Además, el área foliar, perímetro de la hoja medido y longitud de la hoja.

\section{RESULTADOS Y DISCUSIÓN}

El pH varió entre 6,9 a 7,8 (Cuadro 2) y es en el tratamiento "B" donde el porcentaje de lombrihumus era del $30 \%$, este es el rango donde mejor se desarrollan las plantas (Rodríguez 1987).

La Capacidad de Intercambio Catiónico, en miliequivalentes por cada $100 \mathrm{~g}$ de solución, fue alto en todos los tratamientos (Cuadro 2). Existió una relación directa de equilibrio entre los cationes de la solución del suelo y los del complejo absorbente de las plantas, ya que los valores presentados en calcio y magnesio fueron altos (Rodríguez et al. 1992).

En el tratamiento "D" los valores de materia orgánica fueron elevados $(9,4)$ y según Ortiz (1990), la descomposición de materia orgánica se debe a que se producen diferentes nutrimentos, necesarios todos ellos para el desarrollo de las plantas. Por lo general, estos nutrientes son liberados y pueden satisfacer las necesidades de los vegetales.

Los valores de nitrógeno al ser altos, favorecen el crecimiento vegetativo tanto en la parte radical como caulinar. El nitrógeno tiene un importante papel en el alargamiento y en la ramificación de las raíces (Simpson 1998), proceso que favoreció el enraizamiento e indirectamente en el prendimiento del injerto in situ de la estaca.

Los valores para el fósforo fueron similares en todos los tratamientos, y altos en todas las mezclas. En suelos con pH de 6,0 a 7,0 el fósforo disponible en el sustrato es más aprovechable (Ortiz 1990).

A pesar de que los valores de potasio resultaron bajos en todos los tratamientos, Rodríguez et al. (1992) menciona que el pH del suelo influye en su retención en el complejo absorbente. En suelos neutros y ligeramente alcalinos, el potasio es más retenido por las micelas de éste. Aunque exista potasio las plantas encuentran la cantidad necesaria por: a) Una constante renovación y desplazamiento del Potasio hacía la solución y b) porque las plantas también lo absorben por "contacto" con el complejo absorbente del suelo, situación que se presentó en las mezclas.

Para el Factor "A" (fechas de estacado e injertación), resultaron significativas las siguientes variables: longitud de planta, número de nudos, hojas por planta, área foliar, perímetro de hojas y longitud de hoja (Cuadro 3). La interacción de los dos factores A y B, resultó ser significativa para longitud de planta, diámetro de tallo y hojas por planta, y altamente significativo para área foliar, perímetro y longitud de hoja.

Cuadro 2. Determinación de la fertilidad de los sustratos empleados en el injerto in situ de Prunus salicina. Facultad de Ciencias Agrícolas de la Universidad Autónoma del Estado de México (FCA-UAEM), México. 2003.

\begin{tabular}{ccccccccccccccc}
\hline Sustrato & $\mathbf{p H}$ & $\begin{array}{c}\text { CIC meq/ } \\
\mathbf{1 0 0} \mathbf{g}\end{array}$ & $\begin{array}{c}\text { Mat. } \\
\text { Org. }\end{array}$ & $\begin{array}{c}\text { Ca. } \\
\mathbf{I n t} .\end{array}$ & $\begin{array}{c}\mathbf{M g} \\
\mathbf{p p m}\end{array}$ & $\begin{array}{c}\mathbf{N a} \\
\mathbf{p p m}\end{array}$ & $\begin{array}{c}\mathbf{C} \\
(\boldsymbol{\%})\end{array}$ & $\begin{array}{c}\mathbf{N} \\
(\boldsymbol{\%})\end{array}$ & $\begin{array}{c}\mathbf{P} \\
\mathbf{p p m}\end{array}$ & $\begin{array}{c}\mathbf{K} \\
\mathbf{p p m}\end{array}$ & $\begin{array}{c}\mathbf{M n} \\
\mathbf{p p m}\end{array}$ & $\begin{array}{c}\mathbf{F e} \\
\mathbf{p p m}\end{array}$ & $\begin{array}{c}\mathbf{B} \\
\mathbf{m g}\end{array}$ & $\begin{array}{c}\mathbf{C u} \\
\mathbf{m g}\end{array}$ \\
\hline $\mathbf{A}$ & 6,9 & 37,5 & 3,7 & 35,9 & 61,6 & 35,9 & 2,1 & 0,24 & 41 & 1,8 & 0,58 & 2,2 & 0,003 & 12,0 \\
$\mathbf{B}$ & 7,2 & 31,2 & 7,1 & 39,9 & 80,1 & 39,9 & 4,1 & 0,31 & 35 & 2,3 & 0,56 & 12,0 & 0,004 & 12,0 \\
$\mathbf{C}$ & 7,3 & 31,2 & 5,0 & 51,8 & 76,1 & 51,8 & 2,9 & 0,36 & 34 & 2,5 & 0,34 & 0,6 & 0,007 & 12,0 \\
$\mathbf{D}$ & 7,8 & 37,5 & 9,4 & 33,9 & 76,1 & 36,9 & 5,5 & 0,21 & 35 & 3,2 & 0,28 & 0,33 & 0,002 & 0,78 \\
\hline
\end{tabular}

Fuente: Laboratorio de Suelos de la Facultad de Ciencias Agrícolas de la Universidad Autónoma del Estado de México. 2002. 
Cuadro 3. Análisis de varianza de las variables medidas en el experimento de evaluación de sustratos con lombrihumus en la producción de plántulas de ciruelo. Facultad de Ciencias Agrícolas de la Universidad Autónoma del Estado de México (FCA-UAEM), México. 2003.

\begin{tabular}{|c|c|c|c|c|c|c|c|c|c|}
\hline $\begin{array}{l}\text { Fuente de } \\
\text { variación }\end{array}$ & $\begin{array}{c}\text { Grados de } \\
\text { libertad }\end{array}$ & $\begin{array}{c}\text { Longitud } \\
\text { de planta } \\
(\mathrm{cm})\end{array}$ & $\begin{array}{c}\text { Diámetro } \\
\text { de tallo }\end{array}$ & $\begin{array}{l}\text { Número } \\
\text { de nudos }\end{array}$ & $\begin{array}{l}\text { Porcentaje de } \\
\text { prendimiento }\end{array}$ & $\begin{array}{c}\text { Hojas por } \\
\text { planta }\end{array}$ & $\begin{array}{l}\text { Área } \\
\text { foliar } \\
\left(\mathrm{cm}^{2}\right)\end{array}$ & $\begin{array}{c}\text { Perímetro } \\
\text { de hojas } \\
(\mathrm{cm})\end{array}$ & $\begin{array}{l}\text { Longitud } \\
\text { de hojas } \\
\text { (cm) }\end{array}$ \\
\hline Fecha & 1 & $0,028 *$ & $0,086 \mathrm{NS}$ & $0,036^{*}$ & $0,358 \mathrm{NS}$ & $0,014 *$ & $0,148^{*}$ & $0,014 *$ & $0,038^{*}$ \\
\hline Sustrato & 3 & $0,508 \mathrm{NS}$ & $0,182 \mathrm{NS}$ & $0,943 \mathrm{NS}$ & $0,011 *$ & $0,439 \mathrm{NS}$ & $0,039 \mathrm{NS}$ & $0,081 \mathrm{NS}$ & $0,054 \mathrm{NS}$ \\
\hline $\begin{array}{l}\text { Fecha x sus- } \\
\text { trato }\end{array}$ & 3 & 0,027 * & $0,020 *$ & $0,100 \mathrm{NS}$ & $0,106 \mathrm{NS}$ & $0,015^{*}$ & $0,009 * *$ & $0,001 * *$ & $0,009 * *$ \\
\hline Error & 24 & & & & & & & & \\
\hline Total & 31 & & & & & & & & \\
\hline
\end{tabular}

NS =No significativo $; *$ Significativo $; *$ =Altamente significativa

Al analizar la comparación de medias (Cuadro 4) la fecha óptima para el estacado e injertación en la mayoría de los casos fue el 15 de enero. En el mes de diciembre sólo la variable longitud de hoja obtuvo los mejores resultados.

Después de haber trabajado con estacas del cultivar Mariana 2624, se verificó, en algunos estudios, que éstas poseen una capacidad rizógena importante cuando las estacas son preparadas en el período adecuado (Baldini et al. 1990). Para el estudio que nos ocupa y en las condiciones ambientales de Toluca, México, fue el mes de enero, de tal manera que habiendo enraizado las estacas, el crecimiento vegetativo fue favorecido.

En el mes de enero se obtuvieron los mejores valores para longitud de planta, número de nudos, hojas por planta y perímetro de hojas (Cuadro 3), probablemente las condiciones ambientales presentes favorecieron el crecimiento y desarrollo de la porción aérea de la estaca. En el periodo invernal los días en enero se mantienen soleados, proceso que favoreció la fotosíntesis y el incremento en longitud de tallo, la existencia de un mayor número de nudos y consecuentemente un mayor número de hojas, aunque estas no fueran tan largas.

Varios autores también observaron que la época en que se realiza la obtención de las estacas, tiene gran influencia en la capacidad de enraizamiento (Dutra et al. 2002, Kersten et al. 1993, Sharma y Air 1989).

En P. salicina Lindl., la fecha de colecta de las estacas es decisiva para su enraizamiento, considerando además los diferentes cultivares que se manejen (Seganfredo et al. 1997).

Es conveniente recordar que el porcentaje de prendimiento hace referencia a la unión del injerto con el patrón, de tal forma que las dos porciones se unan para continuar el crecimiento como una sola planta; la porción inferior producirá el sistema radical y la superior el caulinar con las características de la variedad seleccionada.

Cuadro 4. Comparación de medias de las dos fechas de siembra (15 de diciembre 2002 y 15 de enero 2003) según Tukey con $\alpha=0,05$ para las variables medidas. Facultad de Ciencias Agrícolas de la Universidad Autónoma del Estado de México (FCA-UAEM), México. 2003.

\begin{tabular}{lccccc}
\hline Fechas & $\begin{array}{c}\text { Longitud de } \\
\text { planta }(\mathbf{c m})\end{array}$ & $\begin{array}{c}\text { Número de } \\
\text { nudos }\end{array}$ & $\begin{array}{c}\text { Hojas por } \\
\text { planta }\end{array}$ & $\begin{array}{c}\text { Perímetro de } \\
\text { hojas }(\mathbf{c m})\end{array}$ & $\begin{array}{c}\text { Longitud de } \\
\text { hojas }(\mathbf{c m})\end{array}$ \\
\hline Diciembre & $1,09 \mathrm{~b}$ & $57,59 \mathrm{~b}$ & $45,01 \mathrm{~b}$ & $758,76 \mathrm{~b}$ & $356,38 \mathrm{a}$ \\
Enero & $1,22 \mathrm{a}$ & $67,49 \mathrm{a}$ & $51,01 \mathrm{a}$ & $852,15 \mathrm{a}$ & $314,90 \mathrm{~b}$ \\
\hline
\end{tabular}

Medias con la misma literal en las filas no difieren significativamente. 
El enraizamiento del patrón depende de la elección de la estaca y su preparación, las condiciones del medio donde se coloca y la época del estacado (Cuisance 1988)

Se recomienda que el sustrato para el enraizado deba de permitir la posición vertical de la estaca, estar constituido por materiales que permitan una buena aireación y drenaje, por lo que se recomienda usar sustratos orgánicos (Vezzosi 1999).

El porcentaje de prendimiento (Figura 1) estuvo directamente relacionado con el tipo de estaca que se selecciona y como se mencionó en la metodología, se eligieron estacas del ciclo vegetativo anterior. Esto, aunado a un $\mathrm{pH}$ del suelo casi neutro; a la disponibilidad de nutrientes de la mezcla, y a una humedad constante permite la formación de un sistema radicular que le conferirá a la futura planta un sólido anclaje (Simpson 1998).

En un trabajo realizado por Pasinato et al. (1998), en donde se probaron diferentes cultivares de $P$. salici$n a$, el que presentó mayor porcentaje de enraizamiento fue el cultivar Roxa de Itaquera con un 76,1\% seguido de la variedad Ace y Sangal con $50,7 \%$ y 48,1\%. En este trabajo sólo se midió el porciento de enraíce en campo sin considerar la injertación in situ.

Cuando Rossal et al. (1997) realizaron un estudio para verificar en qué época del año en Brasil se presenta la mayor acumulación de triptofano en las estacas; ya que este se emplea como cofactor de enraizamiento y precursor de auxinas, encontraron que este cofactor se presentó en el período del 14 al 28 de septiembre, indicando que no es necesaria la aplicación de reguladores de crecimiento externo para que las estacas puedan generar raíces (Tomer y Kumar 1989).

Se considera que el porcentaje de prendimiento fue relativamente alto ya que superó el $50 \%$ en dos de las mezclas y por lo que respecta al área foliar, influyó no sólo en el crecimiento, sino en el desarrollo de las hojas (Figura 1).

Con los análisis foliares realizados a las diferentes muestras (Cuadro 5), se puede verificar que en ningún caso se presentaron carencias ni de macro ni de micro nutrientes, ya que fueron proporcionados por cada una de las mezclas en los tratamientos (Simpson 1998). Por lo que se podría considerar que para el caso del sustrato "B" con $40 \%$ de turba, $30 \%$ de perlita y $30 \%$ de lombrihumus, las plantas mostraron mayor asimilación de nutrimentos y por ello el mayor de los valores para el área foliar.

La comparación de medias (Cuadro 6), nos muestra que para el 15 de diciembre el sustrato "C", presentó los mejores resultados en diámetro de tallo y porcentaje de prendimiento, y el sustrato "B" para la longitud de hojas. No existieron diferencias entre los sustratos para longitud y hojas por planta, área foliar y perímetro de la hoja. En diciembre el mejor resultado

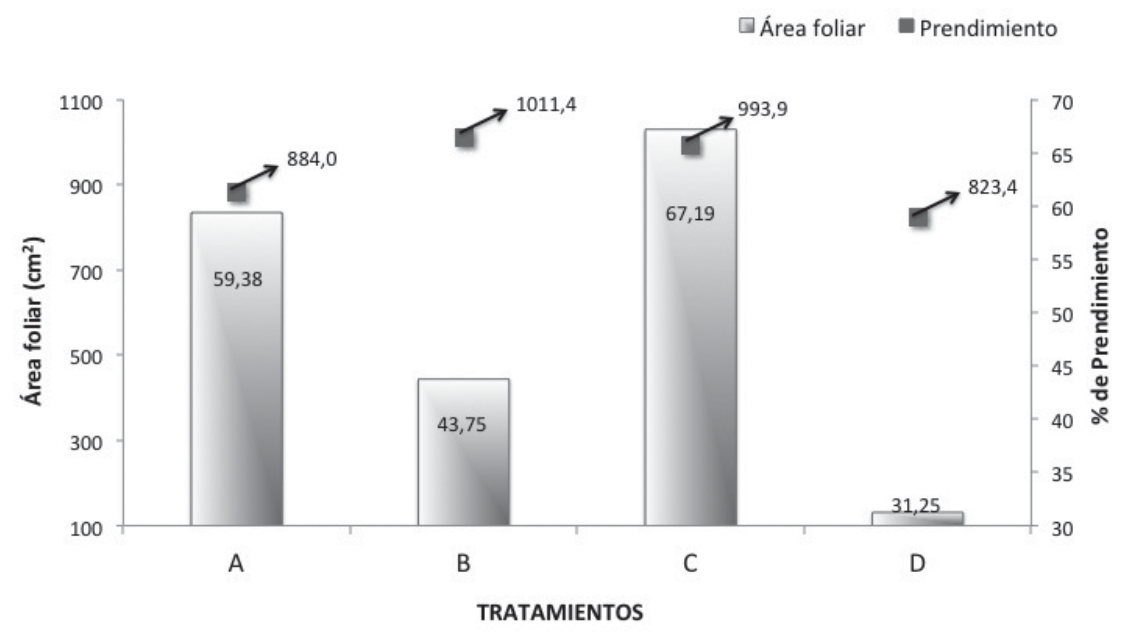

Figura 1. Área foliar y \% de prendimiento de las plantas de ciruelo logradas en los diferentes sustratos. Facultad de Ciencias Agrícolas de la Universidad Autónoma del Estado de México (FCA-UAEM), México. 2003. 
Cuadro 5. Análisis foliar de las plantas de ciruelo obtenidas por injertación considerando fechas y sustrato. Facultad de Ciencias Agrícolas de la Universidad Autónoma del Estado de México* (FCA-UAEM), México. 2003.

\begin{tabular}{lccccccccc}
\hline $\begin{array}{c}\text { Fecha } \\
\text { X Sustrato }\end{array}$ & $\begin{array}{c}\text { N } \\
\text { Total \% }\end{array}$ & $\begin{array}{c}\text { F } \\
\text { Total \% }\end{array}$ & $\begin{array}{c}\text { P } \\
\text { Total \% }\end{array}$ & $\begin{array}{c}\text { Calcio } \\
\text { Total \% }\end{array}$ & $\begin{array}{c}\text { Mg } \\
\text { Total \% }\end{array}$ & $\begin{array}{c}\text { Sodio } \\
\text { Total \% }\end{array}$ & $\begin{array}{c}\text { Fierro } \\
\text { Total \% }\end{array}$ & $\begin{array}{c}\text { Mn } \\
\text { Total \% }\end{array}$ & $\begin{array}{c}\text { Boro } \\
\text { Total \% }\end{array}$ \\
\hline $\mathbf{A}^{\mathbf{1}}$ Dic & 2,93 & 0,20 & 1,83 & 1,14 & 0,37 & 0,06 & 93,34 & 47,07 & 1,69 \\
A Ene & 2,95 & 0,17 & 2,16 & 1,08 & 0,31 & 0,07 & 109,05 & 62,07 & 1,93 \\
B Dic & 3,32 & 0,26 & 1,77 & 0,98 & 0,46 & 0,04 & 77,12 & 48,20 & 1,71 \\
B Ene & 2,97 & 0,24 & 1,72 & 0,78 & 0,57 & 0,04 & 58,79 & 70,33 & 1,69 \\
C Dic & 3,03 & 0,14 & 1,85 & 0,84 & 0,29 & 0,07 & 81,27 & 51,60 & 2,01 \\
C Ene & 3,06 & 0,13 & 2,07 & 0,92 & 0,04 & 0,08 & 90,92 & 125,59 & 1,91 \\
D Dic & 3,32 & 0,25 & 2,33 & 0,94 & 0,52 & 0,06 & 94,98 & 61,06 & 1,91 \\
D Ene & 3,05 & 0,22 & 2,22 & 0,67 & 0,39 & 0,05 & 60,85 & 74,37 & 1,81 \\
\hline
\end{tabular}

* Laboratorio de Suelos de la Secretaría de Desarrollo Agropecuario - Gobierno del Estado de México. Toluca México. Octubre 2003. ${ }^{1} \mathrm{~A}=60 \%$ de Turba, $30 \%$ de Perlita, $10 \%$ de lombrihumus; $\mathrm{B}=40 \%$ de Turba, $30 \%$ de Perlita, $30 \%$ de lombrihumus; $\mathrm{C}=20 \%$ de Turba, $30 \%$ de Perlita, $50 \%$ de lombrihumus; $\mathrm{D}=70 \%$ de Turba, $30 \%$ de Perlita.

Cuadro 6. Comparación de medias de la interacción de dos fechas de estacado e injertación en ciruelo y cuatro sustratos en dos fechas de estacado e injertación ( 15 de diciembre 2002 y 15 de enero 2003) según el método de Tukey con $\alpha=0,05$ para las variables medidas. Facultad de Ciencias Agrícolas de la Universidad Autónoma del Estado de México (FCA-UAEM), México. 2003.

\begin{tabular}{|c|c|c|c|c|c|c|c|c|}
\hline Periodo & Tratamiento & $\begin{array}{l}\text { Longitud } \\
\text { de planta }\end{array}$ & $\begin{array}{c}\text { Diámetro } \\
\text { de tallo }\end{array}$ & $\begin{array}{l}\text { Porcentaje de } \\
\text { prendimiento }\end{array}$ & $\begin{array}{c}\text { Hojas por } \\
\text { planta }\end{array}$ & $\begin{array}{l}\text { Área } \\
\text { foliar }\end{array}$ & $\begin{array}{c}\text { Perímetro } \\
\text { de hojas }\end{array}$ & $\begin{array}{r}\text { Longitud } \\
\text { de hojas }\end{array}$ \\
\hline Diciembre & $\mathrm{A}^{1}$ & 1,14 ba* $^{*}$ & 6,19 ba & $65,63 \mathrm{ba}$ & 48,24 ba & 922,82 ba & $\begin{array}{c}822,47 \\
\text { bac }\end{array}$ & $300,83 \mathrm{~b}$ \\
\hline Diciembre & B & $1,04 \mathrm{ba}$ & $5,85 \mathrm{ba}$ & $50,00 \mathrm{ba}$ & $43,47 \mathrm{~b}$ & $842,57 \mathrm{~b}$ & $750,85 \mathrm{bc}$ & $446,31 \mathrm{a}$ \\
\hline Diciembre & $\mathrm{C}$ & 1,14 ba & $7,22 \mathrm{a}$ & $81,25 \mathrm{a}$ & 48,55 ba & $924,84 \mathrm{ba}$ & $\begin{array}{c}843,49 \\
\text { bac }\end{array}$ & $315,00 \mathrm{~b}$ \\
\hline Diciembre & $\mathrm{D}$ & $0,93 \mathrm{~b}$ & $4,20 \mathrm{~b}$ & $18,75 \mathrm{~b}$ & $39,75 \mathrm{~b}$ & 791,02 b & $618,22 \mathrm{c}$ & $363,38 \mathrm{ba}$ \\
\hline Enero & A & $1,15 \mathrm{ba}$ & $6,33 \mathrm{ba}$ & $53,13 \mathrm{ba}$ & 47,75 ba & 845,17 b & $\begin{array}{c}792,65 \\
\text { bac }\end{array}$ & $352,26 \mathrm{ba}$ \\
\hline Enero & B & $1,29 \mathrm{a}$ & $6,84 \mathrm{a}$ & $37,50 \mathrm{ba}$ & 58,74 a & $1180,34 \mathrm{a}$ & 1010,07 a & $321,02 \mathrm{ba}$ \\
\hline Enero & $\mathrm{C}$ & $1,17 \mathrm{ba}$ & $6,22 \mathrm{ba}$ & 53,13 ba & $45,00 \mathrm{ba}$ & $862,99 \mathrm{~b}$ & $733,49 \mathrm{bc}$ & $319,33 \mathrm{~b}$ \\
\hline Enero & $\mathrm{D}$ & $1,26 \mathrm{ba}$ & $6,81 \mathrm{a}$ & $43,75 \mathrm{ba}$ & $52,52 \mathrm{ba}$ & $855,88 \mathrm{~b}$ & 872,36 ba & $266,97 \mathrm{~b}$ \\
\hline
\end{tabular}

* Medias con la misma literal no difieren significativamente.

${ }^{1} \mathrm{~A}=60 \%$ de Turba, $30 \%$ de Perlita, $10 \%$ de lombrihumus; $\mathrm{B}=40 \%$ de Turba, $30 \%$ de Perlita, $30 \%$ de lombrihumus; $\mathrm{C}=20 \%$ de Turba, $30 \%$ de Perlita, $50 \%$ de lombrihumus; $\mathrm{D}=70 \%$ de Turba, $30 \%$ de Perlita. 
se obtuvo con el sustrato $\mathrm{C}$ formado con $20 \%$ de turba, $30 \%$ de perlita y $50 \%$ de lombrihumus. Para el 15 de enero con el sustrato "B" en diámetro de tallo, hojas por planta y perímetro de hojas longitud de la planta y área foliar, se presentaron los mejores resultados en la parte área. El mayor porcentaje de prendimiento se presentó en el sustrato $\mathrm{C}$ con $50 \%$ de lombrihumus que aunque no difiere del sustrato A, las hojas alcanzaron mayor área foliar En este sentido se considera que el área foliar es determinante para que el desarrollo de la planta alcance su óptimo.

\section{LITERATURA CITADA}

Acosta, MA. 2005. Revista Extra 2005, p. 62. (en línea). Consultado 14 set. 2010. Disponible en http://www. horticom.com/revistasonline/extra05/A_Acosta.pdf

Baldini, E; Escaramuzzi, F. 1990. Il susino. Editorial REDA. Università di Bologna e Firenze. p. 27-35.

Calderón, AE. 1998. Fruticultura general. ECA. 5 ed. México, DF. p. 642-643.

Cruz, NM; Cruz, CJ; Rigel, CI. 1998. Propagación de la macadamia por injertación y estacado. In Memoria Fundación Salvador Sánchez Colín CICTAMEX. Coatepec Harinas, México. p. 82-89.

Cuisance, P. 1988. La multiplicación de las plantas y el vivero. Editorial Mundi - Prensa. Madrid, España. p. 165.

De Sanzo, C; Ravera, A. 1999. Cómo crear lombrices rojas californianas. Programa de Autosuficiencia Regional. (en línea). Provincia de Buenos Aires, Argentina. Consultado 11 jul. 2003. Disponible en http://www. visitweb.com/lombriz

Dutra, LF; Kersten, E; Fachinello, JC. 2002. Época de coleta, ácido indolbutírico e triptofano no enraizamento de estacas de pessegueiro. Sci. agric. 59(2):327333 (en línea). Consultado 8 ago. 2003. Disponible en http://www.scielo.br/scielo.php?pid=S010390162002000200019\&script=sci_abstract\&tlng=pt

García, CN. 2000. Manejo del suelo y de la materia orgánica en los agroecosistemas. In Martínez, C; Rámirez, I. eds. Lombricultura y agricultura sustentable. Editorial Futura. SAGAR, Texcoco, México. p. 25-28.

García, E. 1988. Modificaciones al sistema de clasificación climática de Köppen Instituto de Geografía, UNAM México, D.F. p. 135-137.

Grenón, CG. 2001. Antología curso de propagación de ornamentales. DGETA. Facultad de Ciencias Agrícolas, UAEMEX. p. 15-18.
Grenón, CG; González, CA. 2008. Elaboración de compostas y lombricultura. Apuntes del curso-taller de Educación Continua 2008. Facultad de Ciencias Agrícolas, UAEMex. Toluca, México. p. 55.

Guerrero, PV. 1998. Introducción a la agricultura orgánica. In CIAD ed. Memorias del Simposium: Agricultura Orgánica y de Baja Residualidad. Cd. Cuauhtémoc, Chih., México. p. 45-51.

Hartmann, HT; Kester, DE. 1999. Propagación de plantas. Principios y prácticas. Editorial Continental, S.A. México. p. 274-280; 474-477 y 678-680. (en línea). Consultado 11 de octubre 2011. Disponible http:// www.forestnutrition.org/espanol/leafarea.htm

Kersten, E; Luchéis, AA; Gutiérrez, LE. 1993. Efeito do ácido indolbutirico no enraizamento de estacas de ramos de plantas de amexeira (Prunus salicina Lindl). Scientia Agrícola. Piracicaba 50(1):19-26.

Mirabelli, E. 1995. Curso intensivo de lombricultura. Ed. Facultad de Agronomía, Universidad de Buenos Aires, Argentina. p. 9-10. (en línea). Consultado 23 feb. 2002. Disponible en http://www.dqingenieria.com/ abono/images/lombrices/lombricesrojas.pdf

Ortiz, VB; Ortiz, SC. 1990. Edafología. Universidad Autónoma de Chapingo. p. 291.

Pasinato, V; Nachtigal, JC; Kersten, E. 1998. Enraizamento de estacas lenhosas de cultivares de Amexeira (Prunus spp.) em condicões de campo. Sci. Agric. 55(2):265-268.

Rodríguez, N; Ramírez, SLF; Sustaita, RF. 1992. Materia orgánica. Suelo e influencia directa en la planta. Universidad Autónoma de Chapingo. Dpto. de Suelos. Texcoco, México. p. 1-21.

Rodríguez, SF. 1987. Fertilizantes. Nutrición vegetal. A. G. T. Editor, S. A. México. p. 157.

Rossal, PAL; Kersten, E; Conter, PF. 1997. Estudo comparativo da evolucão do nível de triptofano em ramos de amexeira (Prunus salicina, Lind1). Scientia Agricola 54:174-177.

Sandhawalia, JS. Bal. 1996. Acta Hort. 517 de ISHS. XXV Congreso Hortícola Internacional (en línea). Consultado 11 jul. 2003. Disponible en http://www. actahort.org/books/517/51

Seganfredo, R; Nachtigal, C; Kersten, E. 1997. Infuêcia do ácido indolbutirico e de épocas de colecta de estacas no enraizamiento de cultivares de Amexeira (Prunus salicina Lindl). Rev. Brásileira de Agrociencia 1(1):40-42.

Sharma, SD; Aier, NB. 1989. Seasonal rooting behaviour of cuttings of plum cultivars as influenced by IBA Treatments. Scientia Horticulturae 40:297-303.

Simpson, K. 1998. Abonos y estiércoles. Editorial ACRIBIA, S. A. Zaragoza. p. 155. 
Szecsko, V; Csikos, A; Hrotko, K. 2001. Timing of hardwood cuttings in the propagation of rootstocks. In Proceedings of $9^{\text {th }}$ International Conference of Horticulture, September $3^{\text {th }}-6^{\text {th }}$. Lednice, Czech Republic, Volumen 1, p. 227-232.
Tomer, NS; Kumar, H. 1989. Extent of the possibilities of propagation different plum cultivars by cuttings. Indian Journal of Horticulture 37:369-370.

Vezzosi, C. 1999. Vivaistica ornamentale. EDAGRICOLE, Bologna, Italia. p. 280. 